\title{
Cancer diagnosis disclosure from Portuguese physicians
}

\author{
LUCÍLIA OLIVEIRA, M.D., MARTA FERNANDES, M.D., ZULMIRA SANTOS, M.D., \\ HUGO BASTOS, M.D., AND ANA CABRAL, M.D. \\ Department of Psychiatry, Psycho-Oncology Unit, Coimbra University Hospital Centre, Coimbra, Portugal \\ (RECEIVED January 20, 2014; ACCEPTED June 19, 2014)
}

\begin{abstract}
Objective: Doctor-patient communication in oncology, particularly concerning diagnostic disclosure, is a crucial factor related to the quality of the doctor-patient relationship and the psychological state of the patient. The aims of our study were to investigate physicians' opinions and practice with respect to disclosure of a cancer diagnosis and to explore potential related factors.

Method: A self-report questionnaire developed for our study was responded to by 120 physicians from Coimbra University Hospital Centre and its primary healthcare units.

Results: Some $91.7 \%$ of physician respondents generally disclosed a diagnosis, and $94.2 \%$ were of the opinion that the patient knowing the truth about a diagnosis had a positive effect on the doctor-patient relationship. A need for training about communicating with oncology patients was reported by $85.8 \%$ of participants. The main factors determining what information to provide to patients were: (1) patient intellectual and cultural level, (2) patient desire to know the truth, and (3) the existence of family.

Significance of results: Our results point to a paradigm shift in communication with cancer patients where disclosure of the diagnosis should be made part of general clinical practice. Nevertheless, physicians still experience difficulties in revealing cancer diagnoses to patients and often lack the skills to deal with a patient's emotional responses, which suggests that more attention needs to be focused on communication skills training programs.
\end{abstract}

KEYWORDS: Cancer disclosure, Portugal, Communication skills, Doctor-patient relationship

\section{INTRODUCTION}

The quality of communication is an essential feature of the doctor-patient relationship, which is one of the most crucial issues in healthcare. A doctor's communication skills are probably most important when informing a patient about a diagnosis of oncological disease and its prognosis. An open and supportive communication exchange at this point may perhaps reduce the psychological impact on the patient. The question about whether to tell the truth to or withhold information from a cancer patient has been a

Address correspondence and reprint requests to: Lucília Oliveira, Department of Psychiatry, Coimbra University Hospital Centre. Praceta Professor Mota Pinto, 3000-075 Coimbra, Portugal. E-mail: lucilia.guimaraes@gmail.com. matter for debate among health professionals for many years. The research on this issue has revealed striking differences among varied countries and cultures.

General medical opinion and practice with regard to this matter has undergone several changes during the last few decades. In the middle of the twentieth century, American physicians preferred not to tell the truth to a patient, but full disclosure became the norm by the 1980s (Oken, 1961; Novack et al., 1979). Changing opinions with regard to social power relationships, interpersonal politics, individual autonomy, and a patient's right to information have contributed to this shift. As a result, the legal and ethical constraints in the American healthcare system now make diagnostic disclosure a statutory obligation. 
Most physicians in Anglo-Saxon countries and Northern Europe now believe that cancer patients should be informed of their diagnosis (Smith \& Swisher, 1998). A survey of 990 Norwegian physicians that evaluated attitudes about informing cancer patients found that the vast majority (81\%) preferred to provide full diagnostic information (Loge et al., 1996). In addition, the ethics manual of the American College of Physicians reinforces the importance of telling the truth based on the principle of patient autonomy. On the other hand, some cultures, like that of Japan, hold the principle of non-malfeasance to be superior to individual autonomy and therefore prioritize the option of avoiding the negative impact of diagnostic disclosure (Snyder \& Leffler, 2005; Mitchell, 2005).

Holland and colleagues (1987) conducted a survey of members of the International Psycho-Oncology Society assessing the cultural impact on the practice of diagnostic disclosure internationally. They defined two main trends in physicians with regard to revealing a diagnosis of cancer to patients: (1) low-fewer

\section{Table 1. Questionnaire}

1. Is it your standard procedure to inform the patient of the diagnosis of cancer?

2. If not, indicate three main reasons to justify this practice.

3. How do you usually feel when you inform a patient about a diagnosis of cancer?

4. What are your fears when communicating a diagnosis?

5. How do you usually feel when you inform the patient about the prognosis?

6. What are your fears when you tell them about that prognosis?

7. Has clinical experience made you change the way you view this issue? If your answer is yes, what was the main reason?

8. Have you ever had a personal experience of cancer?

9. Have you ever followed closely any member of your family with cancer?

10. Did these experiences change the way you look at this issue? If yes, in what sense?

11. What words do you typically use to inform the patient?

12. On what factors does the information that you give to a patient depend?

13. Have you ever had training in communicating with oncology patients?

14. Do you feel the need for training in this area?

15. Do you consider the patient knowing the truth as being positive or negative in terms of:

a. dealing with the disease

b. the doctor-patient relationship

c. tolerance to treatment

d. planning for the future

e. the psychological state of the patient over the course of the disease than $40 \%$ of those surveyed mentioned the term "cancer" (e.g., Africa, France, Hungary, Italy, Japan, Panama, Portugal, and Spain), and (2) high-more than $80 \%$ conveyed the diagnosis and used the term "cancer" (e.g., Austria, Denmark Finland, The Netherlands, New Zealand, Norway, Sweden, and Switzerland).

The Western medical community now emphasizes full and truthful disclosure of a cancer diagnosis or prognosis and respect for patient autonomy as part of accepted ethical practice. However, some nations still harbor resistance to this practice, and doctors there choose to speak euphemistically about the diagnosis or even the malignant nature of the disease (Mystakidou et al., 2004).

This issue has been very little studied in Portugal, so there is a significant paucity of available data. Two decades ago, Santos and colleagues (1994) investigated whether Portuguese doctors told the truth to their patients about their cancer diagnoses. Some $59 \%$ of the 80 physicians interviewed did not normally tell their patients the truth about a diagnosis, and, when they did, they downplayed the malignant nature of the disease and its prognostic implications. Even though most physicians understood that truth telling would have a positive effect on the doctor-patient relationship, fear of the patient's emotional reaction was the main reason given for withholding information. Ferraz \& Castro (2001) also explored the issue of disclosure of a cancer diagnosis in a study involving 45 physicians at a Portuguese oncological centre. Some $71 \%$ of their respondents reported that they had a policy of disclosure when requested by the patient, while the remaining $29 \%$ very rarely (if ever) disclosed diagnostic information. They gave as the reason for this policy a fear that disclosure would cause psychological damage for their patients.

The main aim of our present study was to investigate how physicians' attitudes about cancer diagnosis disclosure have changed during the two decades since Santos. We also intended to explore the factors related to this very important issue.

\section{METHOD}

A 15-item self-administered questionnaire was specifically designed for our study (see Table 1). It was put together by 120 physicians from Coimbra University Hospital Centre and its primary healthcare units. The data were analyzed using the Statistical Package for the Social Sciences (SPPS, v. 21) for Windows. Statistical procedures included descriptive statistics, frequency counts, cross-tabulation, the chi-square test, and linear regression, with the level of significance set at $p<0.05$. 


\section{RESULTS}

Of the 120 physicians who participated in our survey, 86 were female (72\%) and 34 male (28\%). Their ages ranged between 25 and 62 years, with a mean of 29 . Almost half had a qualification in a medical specialty $(46.7 \%)$, while $25 \%$ were certified in surgery and $28.3 \%$ in family medicine (see Figure 1). Some $69.2 \%$ were residents and $30.8 \%$ attending physicians; $15 \%$ were assistants, $29.2 \%$ graduate assistants, and $8 \%$ service chiefs (Figure 2). More than half $(56.7 \%)$ had less than 5 years of clinical experience and $20 \%$ more than 10 years. Most (74.2\%) communicated a cancer diagnosis to patients less than 5 times a month, and $7.5 \%$ did so more than 10 times a month.

We found that $91.7 \%$ of our physicians usually reveal a cancer diagnosis (Figure 3), though whether complete or partial information was transmitted to the patient depended on many factors considered by the physicians, particularly the intellectual/cultural level of the patient, the desire of the patient to know the truth, and the social and familial support available to them (Table 2). The term "tumor" was employed by a majority $(60.8 \%)$ when disclosing to a patient, while only $18.3 \%$ used the term "cancer."

Among the $8.3 \%$ of physicians who usually do not communicate a diagnosis, more than half had a medical specialty. The most-often cited reasons for withholding information were as follows: fear about the reaction of patients receiving bad news; a belief that patients do not really want to know; concern about the psychological impact of the disclosure on patients; and a request by a family member to withhold the diagnosis from a patient.

When physicians had to inform a patient about the diagnosis directly, they reported feeling worried (43.3\%), sad $(24.2 \%)$, anxious $(15 \%)$, and fearful (15\%). Physicians most feared patients' emotional reactions after the consultation during which the diagnosis was disclosed (61.7\%), while many (27.5\%) were concerned about patients' reactions during the consultation (see Figure 4).

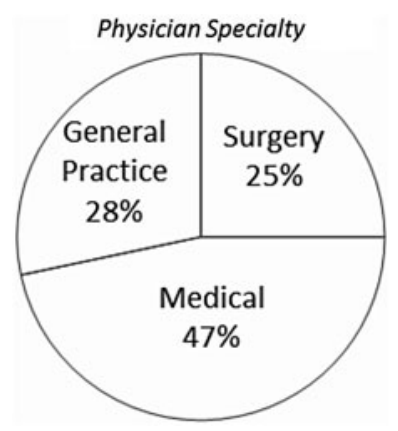

Fig. 1. Distribution according to specialties.
The results related to revelation of a prognosis are similar to those for diagnostic disclosure: physicians reported feeling concern (38.8\%), anxiety (20\%), sadness $(20 \%)$, and fear $(18.3 \%)$. Their main fear was the emotional reaction of patients after $(63.3 \%)$ and during the consultation (20\%). For the majority of physicians (55.8\%), their clinical experiences or having had a relative with cancer were not relevant to how they approached this situation, and there was no statistically significant relationship with level of medical qualification. Some $62.5 \%$ had never had training in how to communicate with cancer patients. In addition, no statistical relationship was found among training and medical specialty, nor among training and number of medical appointments per month where bad news had to be delivered. However, there did appear to be a correlation between training and level of medical career, with residents being better prepared. The need for training in this area was deemed important by $85.8 \%$ of our sample. Answers to the final item on the questionnaire showed that $94.2 \%$ of participants believed a patient's knowledge of their diagnosis would have a positive effect on the doctor-patient relationship. Knowing about the diagnosis was also considered positive by physicians in terms of dealing with the disease (77.5\%), tolerance to treatment (85\%), and planning for the future (79.2\%). The psychological impact of the diagnostic disclosure was considered positive by $56.7 \%$ and physicians, while $35 \%$ expressed the opposite view.

\section{DISCUSSION}

The result that diagnostic disclosure is the norm for $92 \%$ of the physicians who took part in our study says much about what is now standard medical practice in Portugal. This result was higher than those found in other studies where questionnaires were addressed to physicians (Novack et al., 1979; Smith \& Swisher, 1998; Loge et al., 1996), and certainly higher than in some of those carried out in Southern European countries, including Portugal (Santos et al.,

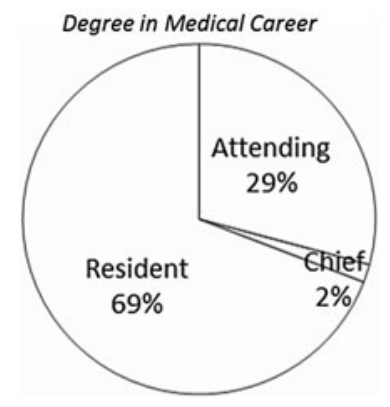

Fig. 2. Distribution according to medical degree. 


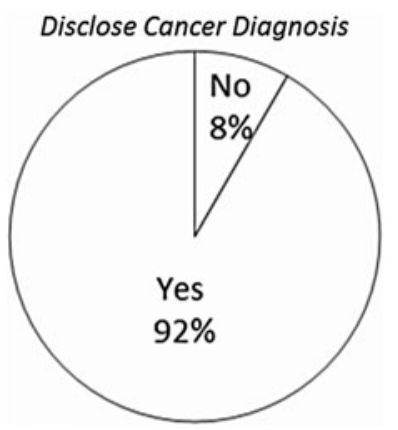

Fig. 3. Percentage of doctors who reveal the diagnosis.

1994; Gonçalves \& Castro, 2001). It reveals a substantial shift in the opinions and attitudes of physicians with regard to telling the truth to cancer patients. Santos and colleagues (1994) reported that only $41 \%$ of physicians believed in truth telling two decades ago, even though there was already the perception that doing so would have a positive effect on the doctor-patient relationship.

Our results reflect an important paradigm shift in the culture of cancer care and practice of Portuguese physicians, from paternalistic disease-focused management to a more humanistic patient-centered approach, in which increasingly more attention is paid to patients' rights to receive truthful and clear information about their clinical status, thus empowering them in making decisions and bolstering their individual autonomy. The clinical practice of medicine in Portugal, as in other countries, was once considered solely from a biomedical standpoint. More

Table 2. Factors that affect which information will be provided to the patient and how

- Intellectual/cultural level (49.2\%)

- The desire of the patient to know the diagnosis $(31.7 \%)$

- Social and family support (25\%)

- Age $(23.3 \%)$

- Prognosis (22.5\%)

- Emotional state of the patient $(20.8 \%)$

- Ability of the patient to understand clinical situation (18.3\%)

- Prior knowledge about the disease $(13.3 \%)$

- Personality characteristics (11.7\%)

- Autonomy and decision-making capacity (8.3\%)

- Stage of disease (6.6\%)

- Having the definitive diagnosis $(6.6 \%)$

- Presence of a family member during the conference $(5.8 \%)$

- The questions the patient asks (5.8\%)

- Adherence to therapy (5\%)

- Socioeconomic level (4.2\%)

- Doctor-patient relationship (4.2\%)

- Will of the family $(2.5 \%)$

- Patient's general condition (2.5\%)

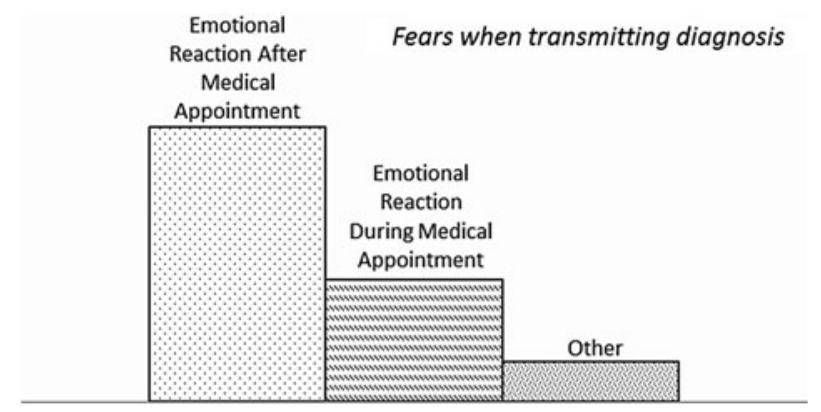

Fig. 4. Physicians' fears when providing a diagnosis.

recently, medical culture has evolved to be more sensitive in recognizing the importance of promoting clear and supportive communication, and thus creating a good therapeutic relationship. Physicians' perceptions, attitudes, and practices with regard to cancer care have undergone quite a change. Most Portuguese physicians now believe that patients have the right to know the truth about their illness, and they are concerned about learning how best to break bad news to patients in a sensitive and humanistic manner, tailoring their approach to each patient's needs, disclosing some truths without destroying all hope, and learning to deal with patients' doubts and uncertainties. They have begun to realize that good doctor-patient communication can improve a patient's clinical outcome, and they are discovering the importance of detecting emotional disturbances in their patients (e.g., depression, anxiety, and adjustment disorders) and referring them to specialists (e.g., psycho-oncologists).

There are many factors behind these changes. Programs that train Portuguese physicians to sharpen their communication skills were developed during the last decade as part of a wider system of psychosocial oncology care projects in an effort to improve cancer care, and also in response to European and international recommendations suggesting that skill communication is a core aspect of the practice of medicine. These programs also envisioned to provide an opportunity to solve a gap in medical education (Grassi et al., 2005). Until then, doctors working with cancer patients were neither required to practice nor taught specific and useful communication skills to help them deal with the difficult moments that occur throughout the disease process that involve giving bad news to patients and families. In addition, advances in the diagnosis and treatment of oncological disease has placed a higher demand on patient cooperation, especially as related to treatment of side effects and tolerance to treatment. The explosion in the dissemination of medical information brought about through globalization, the media, the internet, and social media has also contributed to 
growing demands for high-quality medical services and for recognition of patients' rights to medical information.

Although almost all of our physicians reported diagnostic disclosure to be their norm of practice now, the quantity and quality of information provided to patients still depends on many factors, and our results indicate that patients' intellectual and cultural levels, their desire to know, the family support available to them, and their age are the most important ones for doctors. Though these findings can be instructive, it is important for doctors to avoid any premature assumptions based on these characteristics, and to pay attention to patients' preferences (Fallowfield et al., 2002).

The desire showed by a patient to obtain information was reported as an important factor by $32 \%$ of the doctors in our study in determining whether or not to provide truthful and complete diagnostic information. This number is very close to the $32.5 \%$ found by Santos and colleagues (1994). This attitude may reveal the respect and concern of a doctor for a patient's desire to receive information. However, there is also the risk of ignoring the fact that some patients do not spontaneously express their needs to healthcare professionals. On the contrary, patients often tend to adopt a more passive attitude, waiting for physicians to make the first move, and the doctors can consider this relative passivity a sign of not being interested in receiving information about their disease (Pratt \& Seligman, 1957). Nevertheless, most studies on patient preferences about this matter have revealed that most want to know as much as possible about their illness (diagnosis and prognosis), and prefer to be involved in treatment decisions (Fujimori \& Uchitomi, 2009; Pimentel et al., 1999).

In our study, only $8 \%$ of physicians reported not disclosing diagnostic information as general practice, and they did so primarily for three reasons: (1) fear of the psychological impact; (2) a belief that the patient did not ask for the information and thus had little interest in receiving it; and (3) the families requested that the patient not be told.

Like 20 years ago, the fear of a patient's emotional reaction and concern for their psychological morbidity are still strong factors why physicians are reluctant to disclose diagnostic information. They still reflect the difficulties felt by physicians to communicate cancer diagnoses and prognoses and to deal with difficult emotional states, and thus form the basis for the self-protective attitude formed by the physician (Surbone, 2004). Findings of previous studies on this issue seem contradictory and inconclusive when it comes to the impact of cancer diagnoses and prognoses on a patient's psychological state (Fallowfield et al., 2002; Atesci et al., 2004). Psychological morbidity may be more frequent and serious among patients who were not informed of their diagnosis directly by their physician and had to guess it through the course of their illness. A more closed style of communication has a negative effect on patient adjustment to oncological disease and is a contributing factor in psychopathological outcomes. Psychiatric disorders (a treatable cause of considerable morbidity), poor quality of life, and a worse cancer prognosis can contribute to deepening depression, and $80 \%$ of the suicides in the oncological population are committed by patients with depressive syndromes (Henriksson et al., 1995). It is extremely important to recognize and treat psychiatric morbidity, as well as depression and anxiety.

Family is a third element involved in the communication process. Its influence can be strong enough to condition information as it arrives to the patient, here also depending on social and cultural differences. Some studies have found family resistance to disclosure of information to patients because of fears about the psychological impact on the patient (Miyata et al., 2005; Oksuzoglu et al., 2006).

Our results revealed that more than half of physicians have never received training in communication skills, and those who had were mainly residents. The need for medical training in this area was recognized by $86 \%$ of doctors, which means that in many cases it was felt to be insufficient. Our results demonstrate that the majority of physicians still manifest difficulties at the time of revealing a cancer diagnosis and lack the communication skills to deal with patients' emotional reactions, as illustrated by the answers given by most doctors about their fear and anxiety experienced at the time of diagnostic disclosure. It seems imperative that the level of communication skills training be greatly improved so that doctors can better manage their own emotions and also support their patients emotionally, which would certainly enhance the doctor-patient relationship (Panagopoulou et al., 2008).

\section{CONCLUSION}

Considering the traditional biomedical model that arose from paternalistic and reductionist conceptions of a patient's role in treatment, in which the issue of whether or not to tell the truth was controversial, there has been a paradigm shift in physicians' attitudes about communicating with cancer patients. Our results demonstrate that doctors believe that diagnostic disclosure should be the norm in clinical practice, though they still feel they lack the requisite interpersonal and communicational skills. Without doubt, more attention should be paid to undergraduate and postgraduate medical training in order to 
improve communication between physicians and their patients.

More than a matter of "truth telling," we hope that this issue will turn more into a matter of "truth making" with cancer patients, in the sense of a broader, deeper, and complex paradigm, beyond the sophisticated technology, involving a dynamic therapeutic process where new questions arise and others go on changing through time.

\section{REFERENCES}

Atesci, F.C., Baltalarli, B., Oguzhanoglu, N.K., et al. (2004). Psychiatric morbidity among cancer patients and awareness of illness. Supportive Care in Cancer, $12,161-167$.

Fallowfield, L.J., Jenkins, V.A. \& Beveridge, H.A. (2002). Truth may hurt but deceit hurts more: Communication in palliative care. Palliative Medicine, 16, 297-303.

Ferraz, G.J. \& Castro, S. (2001). Diagnosis disclosure in a Portuguese oncological centre. Palliative Medicine, 15, $35-41$.

Fujimori, M. \& Uchitomi, Y. (2009). Preferences of cancer patients regarding communication of bad news: A systematic literature review. Japanese Journal of Clinical Oncology, 39, 201-216.

Gonçalves, J.F., \& Castro, S. (2001). Diagnosis disclosure in a Portuguese oncological centre. Palliative Medicine, 15, 35.

Grassi, L., Travado, L., Gil, F., et al. (2005). A communication: Intervention for training Southern European oncologists to recognize psychosocial morbidity in cancer patients, I: Development of the model and preliminary results on physicians' satisfaction. Journal of Cancer Education, 20, 79-84.

Henriksson, M.M., Isometsa, E.T., Hietanen, P.S., et al. (1995). Mental disorders in cancer suicides. Journal of Affective Disorders, 36, 11-21.

Holland, J.C., Geary, N., Marchini, A., et al. (1987). International survey of physician attitudes and practice in regard to revealing the diagnosis of cancer. Cancer Investigation, 5, 51-54.
Loge, J.H., Kaasa, S., Ekeberg, O., et al. (1996). Attitudes toward informing the cancer patient: A survey of Norwegian physicians. European Journal of Cancer, 32, 1344-1348.

Mitchell, J.L. (2005). Cross-cultural issues in the disclosure of cancer. Cancer Practice, 6, 153-160.

Miyata, H., Takahashi, M., Saito, T., et al. (2005). Disclosure preferences regarding cancer diagnosis and prognosis: To tell or not to tell? Journal of Medical Ethics, $31,447-451$.

Mystakidou, K., Parpa, E., Tsilila, E., et al. (2004). Cancer information disclosure in different cultural contexts. Supportive Care in Cancer, 12, 147-154.

Novack, D.H., Plumer, R., Smith, R.L., et al. (1979). Changes in physicians' attitudes toward telling the cancer patient. The Journal of the American Medical Association, $241,897-900$.

Oken, D. (1961). What to tell cancer patients: A study of medical attitudes. The Journal of the American Medical Association, 175, 1120-1128.

Oksuzoglu, B., Abali, H., Bakar, M., et al. (2006). Disclosure of cancer diagnosis to patients and their relatives in Turkey: Views of accompanying persons and influential factors in reaching those views. Tumori, 92, 62-66.

Panagopoulou, E., Mintziori, G., Montgomery, A., et al. (2008). Concealment of information in clinical practice: Is lying less stressful than telling the truth? Journal of Clinical Oncology, 26, 1175-1177.

Pimentel, F.L., Ferreira, J.S. \& Vila Real, M. (1999). Quantity and quality of information desired by Portuguese cancer patients. Supportive Care in Cancer, 7, 407-412.

Pratt, L. \& Seligman, A. (1957). Physician's view on the level of medical information among patients. American Journal of Public Health, 47, 1277.

Santos, Z., Santos, G. \& Abrantes, P. (1994). Comunicação médico-doente em oncologia. Acta Medica Portuguesa, 7, 361-365.

Smith, J.T. \& Swisher, K. (1998). Telling the truth about terminal cancer. The Journal of the American Medical Association, 279, 1746-1748.

Snyder, L. \& Leffler, C. (2005). Ethics manual: Fifth edition. Annals of Internal Medicine, 142, 560-582.

Surbone, A. (2004). Persisting differences in truth telling throughout the world. Supportive Care in Cancer, 12, $143-146$ 